\title{
Galois points for a plane curve and its dual curve
}

\author{
Satoru Fukasawa $(*)$ - Kei Miura $(* *)$
}

ABstract - A point $P$ in projective plane is said to be Galois for a plane curve of degree at least three if the function field extension induced by the projection from $P$ is Galois. Further we say that a Galois point is extendable if any birational transformation by the Galois group can be extended to a linear transformation of the projective plane. In this article, we propose the following problem: If a plane curve has a Galois point and its dual curve has one, what is the curve? We give an answer. We show that the dual curve of a smooth plane curve does not have a Galois point. On the other hand, we settle the case where both a plane curve and its dual curve have extendable Galois points. Such a curve must be defined by $X^{d}-Y^{e} Z^{d-e}=0$, which is a famous self-dual curve.

Mathematics Subject Classification (2010). 14H50, 14H05, $12 \mathrm{~F} 10$.

KEYwORDS. Galois point, plane curve, dual curve, self-dual curve.

\section{Introduction}

Let the base field $K$ be an algebraically closed field of characteristic $p=0$ and let $C \subset \mathbb{P}^{2}$ be an irreducible plane curve of degree $d \geq 3$. We recall the notions of dual curve and Galois point.

Let $\mathbb{P}^{2 *}$ be the dual projective plane which parameterizes projective lines of $\mathrm{P}^{2}$ and let $(X: Y: Z),(U: V: W)$ be systems of homogeneous coordinates of $\mathbb{P}^{2}$ and of $\mathbb{P}^{2 *}$ respectively. We denote by $\operatorname{Sing}(C)$ the singular locus of $C$. If $C$ is defined by a homogeneous polynomial $F(X, Y, Z)$,

(*) Indirizzo dell'A.: Department of Mathematical Sciences, Yamagata University, Kojirakawa-machi 1-4-12, Yamagata 990-8560, Japan.

E-mail: s.fukasawa@sci.kj.yamagata-u.ac.jp

The first author was partially supported by JSPS KAKENHI Grant Numbers 22740001, 25800002.

(**) Indirizzo dell'A.: Department of Mathematics, Ube National College of Technology, Ube, Yamaguchi 755-8555, Japan.

E-mail: kmiura@ube-k.ac.jp 
we have a rational map $\gamma=\gamma_{C}: C-\rightarrow \mathbb{P}^{2 *}$, which sends a smooth point $Q \in C \backslash \operatorname{Sing}(C)$ to the point $\left(\frac{\partial F}{\partial X}(Q): \frac{\partial F}{\partial Y}(Q): \frac{\partial F}{\partial Z}(Q)\right) \in \mathbb{P}^{2 *}$ parameterizing the projective tangent line $T_{Q} C$ to $C$ at $Q$. This rational map is called the dual map of $C$ and (the closure of) the image of $C$ is called the dual curve, which is denoted by $C^{*}$. It is well-known that projective duality $C^{* *}=C$ holds (see, for example, [7, 11]).

If the function field extension $K(C) / K\left(\mathbb{P}^{1}\right)$ induced by the projection $\pi_{P}: C-\rightarrow \mathbb{P}^{1}$ from a point $P \in \mathbb{P}^{2}$ is Galois, then the point $P$ is said to be Galois. Moreover, denoting by $G_{P}$ the Galois group associated to the projection $\pi_{P}$, we say that a Galois point $P$ is extendable if any birational transformation of $C$ induced by the Galois group $G_{P}$ can be extended to a linear transformation of $\mathbb{P}^{2}$.

The notion of Galois point was introduced by H. Yoshihara (see e.g. $[4,9,13])$ and it is an interesting topic on plane curves. For instance, a well-known theorem of Noether and later results assure that if $C \subset \mathbb{P}^{2}$ is a smooth curve of degree $d$, then the minimum degree of a morphism $C \rightarrow \mathbb{P}^{1}$ is $d-1$, and all the maps of degree $d-1 \geq 2$ and $d \geq 5$ are projections $\pi_{P}: C \rightarrow \mathbb{P}^{1}$ from some point $P \in C$ and $P \in \mathbb{P}^{2} \backslash C$ respectively (cf. $[3,5,10])$. Thus describing Galois points on a smooth plane curve is equivalent to detect all the Galois coverings $C \rightarrow \mathbb{P}^{1}$ having minimal degrees.

The singular curve defined by $X^{d}-Y^{e} Z^{d-e}=0$, where $e \geq 1$ and $d, e$ are coprime, has lovely properties. Its dual curve is defined by the same equation (up to a projective equivalence, see Lemma 2.5). Therefore, this curve is a "self-dual" curve. It has an (extendable) Galois point $(1: 0: 0)$ (Proposition 2.3) and its dual curve has one, by the self-duality. In the light of this fact, we propose the following problem.

Problem 1.1. If a plane curve has a Galois point and its dual curve has one, what is the curve? Is it a self-dual curve?

In this article, we give an answer. We show the following for smooth curves.

TheORem 1.2. Let $C$ be a smooth plane curve of degree $d \geq 3$. Then, there exist no Galois points for the dual curve of $C$.

By projective duality, we have the following. 
Corollary 1.3. Let $C$ be a plane curve of degree $d \geq 3$ and let $C^{*}$ be the dual curve. If both $C$ and $C^{*}$ have Galois points, then they are singular.

We say that a Galois point $P$ is inner (resp. outer) if $P \in C \backslash \operatorname{Sing}(C)$ (resp. $P \in \mathbb{P}^{2} \backslash C$ ). In the case where Galois points are extendable, we show the following characterization theorems.

THEOREM 1.4. Let $C$ be a plane curve of degree $d \geq 3$ and let $C^{*}$ be the dual curve of $C$.

(I) The following conditions are equivalent.

(1) $C$ and $C^{*}$ have extendable outer Galois points.

(2) $C$ is projectively equivalent to the plane curve defined by $X^{d}-Y^{e} Z^{d-e}=0$ for some $e \geq 1$.

(II) The following conditions are equivalent.

(1) $C$ has an extendable outer Galois point and $C^{*}$ has an extendable inner Galois point.

(2) $C$ and $C^{*}$ have extendable inner Galois points.

(3) $C$ is projectively equivalent to the plane curve defined by $X^{d}-Y^{d-1} Z=0$.

To prove our result we will connect Galois points for a plane curve $C$ and its dual curve. In particular, we will show that - given an extendable Galois point $P$ for $C$ - the Galois group $G_{P}$ has a natural action on the dual curve $C^{*}$, and such an action preserves the fibers of a certain projection $\pi_{\bar{P}}: C^{*} \rightarrow \mathbb{P}^{1}$. Namely,

Proposition 1.5. Let $C$ be a plane curve with an extendable Galois point $P \in \mathbb{P}^{2}$ and let $G_{P}$ be the Galois group. Any $\sigma \in G_{P}$ induces a natural linear transformation $\bar{\sigma}: \mathbb{P}^{2 *} \rightarrow \mathbb{P}^{2 *}$ (see Lemma 2.2 for details). Then, there exists a unique point $\bar{P} \in \mathbb{P}^{2 *}$ such that the map $\sigma \mapsto \bar{\sigma}$ induces an injective homomorphism

$G_{P} \hookrightarrow G[\bar{P}]:=\left\{\tau \in \operatorname{Bir}\left(C^{*}\right) \mid \tau\left(C^{*} \cap \ell \backslash\{\bar{P}\}\right) \subset \ell\right.$ for a general line $\left.\ell \ni \bar{P}\right\}$, where $\operatorname{Bir}\left(C^{*}\right)$ is the group of all birational transformations of $C^{*}$. In particular, the degree of the projection $\pi_{\bar{P}}: C^{*}{ }_{-\rightarrow} \rightarrow \mathbb{P}^{1}$ from $\bar{P}$ is at least the order of $G_{P}$.

In the next Section we will recall some preliminary facts about projective duality and Galois group, and we will achieve Proposition 1.5. Section 3 and Section 4 will be devoted to prove Theorems 1.2 and 1.4 respectively. 


\section{Preliminaries}

Let $C$ be an irreducible plane curve of degree $d \geq 3$. If a point $P \in \mathbb{P}^{2}$ is not in $C$, we define the multiplicity of $C$ at $P$ as zero. We denote by $d^{*}$ the degree of the dual curve $C^{*}$.

We recall duality principle between $\mathbb{P}^{2}$ and $\mathbb{P}^{2 *}$. For a projective line $\ell \subset \mathbb{P}^{2}$, we denote by $[\ell]$ the point in $\mathbb{P}^{2 *}$ corresponding to $\ell$. If $\ell$ is defined by $u X+v Y+w Z=0$ for some $u, v, w \in K$, then the point $[\ell] \in \mathbb{P}^{2 *}$ is given by $(u: v: w)$. For a point $P \in \mathbb{P}^{2}$, we denote by $[P] \subset \mathbb{P}^{2 *}$ the line corresponding to $P$, which parameterizes the star of lines through $P$. If $P$ is defined by $u_{1} X+v_{1} Y+w_{1} Z=u_{2} X+v_{2} Y+w_{2} Z=0$ for some $u_{1}, u_{2}, v_{1}, v_{2}, w_{1}, w_{2} \in K$, then $[P]$ is the line passing through the two points $\left(u_{1}: v_{1}: w_{1}\right),\left(u_{2}: v_{2}: w_{2}\right) \in \mathbb{P}^{2 *}$. Then, we have the following elementary facts.

Lemma 2.1. Let $P \in \mathbb{P}^{2}$ be a point and let $\ell \subset \mathbb{P}^{2}$ be a line. Then, $[[P]]=P$ and $[[\ell]]=\ell$ hold. Furthermore,

$$
P \in \ell \Leftrightarrow[\ell] \in[P]
$$

holds.

Lemma 2.2. Let $\phi$ be a linear transformation of $\mathrm{P}^{2}$ and let $A_{\phi}$ be a matrix representing $\phi$, i.e. $\phi(X: Y: Z)=(X, Y, Z) A_{\phi}$. Then the induced map $\bar{\phi}: \mathbb{P}^{2 *} \rightarrow \mathbb{P}^{2 *} ;[\ell] \mapsto[\phi(\ell)]$ is represented by the transpose of the matrix $A_{\phi}^{-1}$, that is

$$
\bar{\phi}(U: V: W)=(U, V, W){ }^{t} A_{\phi}^{-1} .
$$

Moreover, the diagram

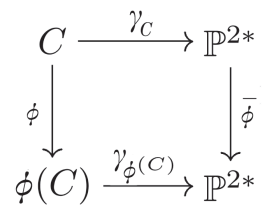

commutes, where the horizontal arrows are the dual maps of $C$ and $\phi(C)$.

We often use the standard form of the defining equation for a plane curve with an extendable Galois point, which is given by the following.

Proposition 2.3 (see $[8,13,15]$ ). Let $P \in \mathbb{P}^{2}$ be a point with multiplicity $m \geq 0$. The point $P$ is extendable Galois for $C$ if and only if there 
exists a linear transformation $\phi$ on $\mathbb{P}^{2}$ such that $\phi(P)=(1: 0: 0)$ and $\phi(C)$ is given by

$$
X^{d-m} G_{m}(Y, Z)+G_{d}(Y, Z)=0,
$$

where $G_{i}(Y, Z)$ is a homogeneous polynomial of degree $i$ in variables $Y, Z$. In this case, the Galois group $G_{\phi(P)}$ is cyclic and there exists a primitive $(d-m)$-th root $\zeta$ of unity such that a generator $\sigma \in G_{\phi(P)}$ is represented by $\sigma(X: Y: Z)=(\zeta X: Y: Z)$.

Firstly, we prove the first assertion of Proposition 1.5 for the curves with the standard form as in Proposition 2.3.

Lemma 2.4. Let $P=(1: 0: 0) \in \mathbb{P}^{2}$, let $C$ be defined by

$$
X^{d-m} G_{m}(Y, Z)+G_{d}(Y, Z)=0,
$$

and let a generator $\sigma \in G_{P}$ be represented by $\sigma(X: Y: Z)=(\zeta X: Y: Z)$. Then, there exists a unique point $\bar{P} \in \mathbb{P}^{2 *}$ such that the map $G_{P} \rightarrow$ $G[\bar{P}] ; \sigma^{i} \mapsto \overline{\sigma^{i}}$ is a well-defined and injective homomorphism.

Proof. By Lemma 2.2, $\bar{\sigma}$ is represented by $\bar{\sigma}(U: V: W)=$ $\left(\zeta^{-1} U: V: W\right)$ and $\bar{\sigma}\left(C^{*}\right)=C^{*}$. Let $\bar{P}=(1: 0: 0) \in \mathbb{P}^{2 *}$. By the representation of $\bar{\sigma}$, we have

$$
\bar{\sigma} \in G[\bar{P}]=\left\{\tau \in \operatorname{Bir}\left(C^{*}\right) \mid \tau\left(C^{*} \cap \ell \backslash\{\bar{P}\}\right) \subset \ell \text { for a general line } \ell \ni \bar{P}\right\} .
$$

We prove the uniqueness of $\bar{P}$. By contradiction we assume that $\bar{\sigma} \in G[\bar{P}] \cap G[Q]$, where $Q \neq \bar{P}$. Let $R \in C^{*}$ be a point not contained in the line passing through $\bar{P}$ and $Q$ such that $\bar{\sigma}(R) \neq R$. By $\bar{\sigma} \in G[\bar{P}] \cap G[Q], \bar{\sigma}(R)$ is contained in the lines passing through $R$ and $\bar{P}, R$ and $Q$ respectively. This implies that $\bar{\sigma}(R)=R$. This is a contradiction.

Proof of Proposition 1.5. By Proposition 2.3, there exists a linear transformation $\phi$ such that $\phi(P)=(1: 0: 0), \phi(C)$ is given by $X^{d-m} G_{m}(Y, Z)+$ $G_{d}(Y, Z)=0$ and a generator $\sigma \in G_{\phi(P)}$ is represented by $\sigma(X: Y: Z)=$ ( $\zeta X: Y: Z)$. By Lemma 2.4, we have an injection $f: G_{\phi(P)} \hookrightarrow G[\overline{\phi(P)}] ; \sigma^{i} \mapsto \overline{\sigma^{i}}$. Let $\bar{P}=\bar{\phi}^{-1}(\overline{\phi(P)})$. We also have isomorphisms $g: G_{P} \rightarrow G_{\phi(P)} ; \tau \mapsto \phi \tau \phi^{-1}$ and $h: G[\overline{\phi(P)}] \rightarrow G[\bar{P}] ; \eta \mapsto \bar{\phi}^{-1} \eta \bar{\phi}$. Since $\left({ }^{t} A_{\phi}^{-1}\right)^{t}\left(A_{\phi}^{-1} A_{\tau} A_{\phi}\right)^{-1}\left({ }^{t} A_{\phi}^{-1}\right)^{-1}=$ $\left({ }^{t} A_{\phi}^{-1}\right){ }^{t} A_{\phi}{ }^{t} A_{\tau}^{-1}{ }^{t} A_{\phi}^{-1}\left({ }^{t} A_{\phi}\right)={ }^{t} A_{\tau}^{-1}$, we have $(h \circ f \circ g)(\tau)=\bar{\tau}$. Therefore, $G_{P} \rightarrow G[\bar{P}] ; \tau \mapsto \bar{\tau}$ is a well-defined and injective homomorphism. The uniqueness of the point $\bar{P}$ follows, similarly to the proof of Lemma 2.4. 
We prove the second assertion. Let $m^{*}$ be the multiplicity of $C^{*}$ at $\bar{P}$. Then, the order of $G[\bar{P}]$ is at most $d^{*}-m^{*}$, which is equal to the degree of the projection $\pi_{\bar{P}}: C^{*}-\rightarrow \mathbb{P}^{1}$. By the first assertion, we have $d-m \leq d^{*}-m^{*}$.

Here, we summarize the properties of the curve defined by $X^{d}-Y^{e} Z^{d-e}=0$.

Lemma 2.5. Let $C$ be defined by $X^{d}-Y^{e} Z^{d-e}=0$. Then, we have the following.

(1) The dual curve $C^{*}$ is given by $(-1)^{d} e^{e}(d-e)^{d-e} U^{d}-d^{d} V^{e} W^{d-e}=0$.

(2) Points $(1: 0: 0) \in \mathbb{P}^{2}$ and $(1: 0: 0) \in \mathbb{P}^{2 *}$ are extendable outer Galois for $C$ and $C^{*}$ respectively.

(3) If $e=d-1$, then points $(0: 1: 0) \in \mathbb{P}^{2}$ and $(0: 1: 0) \in \mathbb{P}^{2 *}$ are extendable inner Galois for $C$ and $C^{*}$ respectively.

Proof. Since the dual map $\gamma$ is given by

$$
\begin{aligned}
& \left(d X^{d-1}:-e Y^{e-1} Z^{d-e}:-(d-e) Y^{e} Z^{d-e-1}\right) \\
= & \left(d X^{d}:-e X Y^{e-1} Z^{d-e}:-(d-e) X Y^{e} Z^{d-e-1}\right) \\
= & \left(d Y^{e} Z^{d-e}:-e X Y^{e-1} Z^{d-e}:-(d-e) X Y^{e} Z^{d-e-1}\right) \\
= & (d Y Z:-e X Z:-(d-e) X Y),
\end{aligned}
$$

we have (1). We have assertions (2) and (3) by Proposition 2.3.

\section{Galois points for the dual curve of a smooth curve}

Let $C \subset \mathbb{P}^{2}$ be a smooth curve of degree $d \geq 3$ and let $C^{*}$ be the dual curve of $C$. The dual map $\gamma: C \rightarrow C^{*}$ is birational ([7], [11, Theorem 1.5.3]) and $C^{*}$ is of degree $d^{*}=d(d-1)$, since the linear system $\left\langle\frac{\partial F}{\partial X}, \frac{\partial F}{\partial Y}, \frac{\partial F}{\partial Z}\right\rangle$ has no base point. Note that any birational transformation of $C$ is extendable when $d \geq 4$ (see [1, Appendix A, 17 and 18] or [2]). Therefore any Galois point is extendable in this case.

LEMma 3.1. Let $C$ be a smooth plane curve of degree $d \geq 4$. Then, any birational map $C^{*} \rightarrow C^{*}$ can be extended to a linear transformation of $\mathbb{P}^{2 *}$. Therefore, any Galois point for $C^{*}$ is extendable. 
Proof. Let $\tau: C^{*}-\rightarrow C^{*}$ be a birational map. Then the rational map $\tau_{0}:=\gamma^{-1} \tau \gamma: C \rightarrow C$ is a birational transformation of $C$. Since $C$ is smooth of degree $d \geq 4, \tau_{0}$ can be extended to a linear transformation of $\mathbb{P}^{2}$ as observed above. Since the diagram

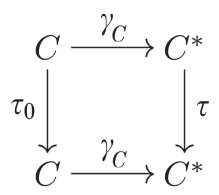

commutes, we have $\left.\overline{\tau_{0}}\right|_{C^{*}}=\tau$. Hence $\tau$ can be extended to a linear transformation of $\mathbb{P}^{2 *}$.

Proof of Theorem 1.2. Let $C$ be a smooth plane curve of degree $d \geq 3$. Throughout we treat the cases $d=3$ and $d \geq 4$ separately.

Firstly, we assume that $d \geq 4$. Aiming for a contradiction, let $R \in \mathbb{P}^{2 *}$ be a Galois point for $C^{*}$, and let $m$ be the multiplicity of $C^{*}$ at $R$. It follows from Lemma 3.1 and Proposition 1.5 that the degree of the projection from $R$ is at most $d$. Therefore, we have $d^{*}-m \leq d$. Since $R \in C^{*}$, there exists a point $R_{0} \in C$ with $\gamma\left(R_{0}\right)=R$. Let $\ell \subset \mathbb{P}^{2 *}$ be a general line with $R \in \ell$. Then, $C^{*} \cap \ell \backslash\{R\}$ consists of exactly $d^{*}-m$ smooth points of $C^{*}$. Since $C^{*} \cap \ell \backslash\{R\}$ consists of only smooth points, there are exactly $d^{*}-m$ points in $\gamma^{-1}\left(C^{*} \cap \ell \backslash\{R\}\right)$ and such points are not flexes (i.e. the intersection multiplicity of $C$ and the tangent line at the point is two). It follows from Lemma 2.1 that $[\ell] \in\left[\gamma\left(Q_{0}\right)\right]=T_{Q_{0}} C \subset \mathbb{P}^{2}$ for any point $Q_{0} \in \gamma^{-1}\left(C^{*} \cap \ell\right)$. This implies that, for a general point $P \in[R]=\left[\gamma\left(R_{0}\right)\right]=T_{R_{0}} C$, there exist exactly $d^{*}-m$ points not in $\gamma^{-1}(R)$ which are not flexes and the tangent lines contain $P$. Then, by Hurwitz formula for the projection $\pi_{P}: C \rightarrow \mathbb{P}^{1}$ from a general point $P \in T_{R_{0}} C \backslash C$, we have $2 g(C)-2+2 \operatorname{deg}\left(\pi_{P}\right)=\operatorname{deg} B$, where $g(C)=\frac{(d-1)(d-2)}{2}$ is the genus of $C$ and $B$ is the ramification divisor. Since the sum of the degrees of $B$ given by points in $C \cap T_{R_{0}} C$ is at most $d-1$, we have $\operatorname{deg} B \leq(d-1)+\left(d^{*}-m\right)$. Thus Hurwitz formula leads to the following inequality

$$
d^{2}-3 d+2 d \leq(d-1)+\left(d^{*}-m\right) \leq 2 d-1 .
$$

This implies that $d \leq 3$. This is a contradiction to the assumption $d \geq 4$.

So we assume that $d=3$. Then, we have $d^{*}=6$. We recall the wellknown fact that there are nine flexes for $C$. Let Flex $(C) \subset C$ be the set of all flexes of $C$. Then $\gamma(\operatorname{Flex}(C))=\operatorname{Sing}\left(C^{*}\right)$ as $C$ does not admit bitangent lines. Firstly we would like to determine lines $\ell \subset \mathbb{P}^{2 *}$ such that 
$\ell \cap \operatorname{Sing}\left(C^{*}\right) \neq \emptyset$. Let $Q \in \operatorname{Flex}(C)$ and let $P \in T_{Q} C$, which is maybe $Q$. Using Hurwitz formula for the projection $\pi_{P}$, the ramification divisor has degree 6 (resp. 4) if $P \neq Q$ (resp. $P=Q$ ). Hence one of the following conditions holds.

(1) There exist three flexes whose tangent lines contain $P$.

(2) There exist exactly two flexes and two other points whose four tangent lines contain $P$.

(3) There exist exactly one flex and four other points whose five tangent lines contain $P$.

(4) $P=Q$ and there exist three points which are not flexes and tangent lines at them contain $P$.

It follows from Lemma 2.1 that one of the following conditions holds if $\ell \cap \operatorname{Sing}\left(C^{*}\right) \neq \emptyset$ for a line $\ell \subset \mathbb{P}^{2 *}$.

(1*) $C^{*} \cap \ell$ consists of three singular points.

(2) $C^{*} \cap \ell$ consists of two singular points and two smooth points.

$\left(3^{*}\right) C^{*} \cap \ell$ consists of one singular point and four smooth points.

(4*) $C^{*} \cap \ell$ consists of exactly one singular point and three smooth points.

Let $R \in \mathbb{P}^{2 *}$ be a point and let $\hat{\pi}_{R}:=\pi_{R} \circ \gamma: C \rightarrow \mathbb{P}^{1}$. We denote by $e_{P}$ the ramification index at $P \in C$ for $\hat{\pi}_{R}$. Note that if $Q \in \operatorname{Flex}(C)$ and $\gamma(Q) \neq R$, then $\hat{\pi}_{R}$ is ramified at $Q$, since the differential of $\gamma$ at $Q$ is zero. The above conditions represent types of ramification indices of flexes for $\hat{\pi}_{R}$. For example, condition $\left(1^{*}\right)$ implies that $e_{Q_{1}}=e_{Q_{2}}=e_{Q_{3}}=2$, where $\gamma\left(Q_{1}\right), \gamma\left(Q_{2}\right), \gamma\left(Q_{3}\right) \in C^{*} \cap \ell$ if $R \in \ell$ and $R \notin C^{*}$.

Assume by contradiction that $R \in \mathbb{P}^{2 *}$ is a Galois point for $C^{*}$. Then, the field extension induced by $\hat{\pi}_{R}$ is Galois. We often use the property of Galois extensions that the ramification index $e_{Q_{1}}$ is equal to the one $e_{Q_{2}}$ if $\hat{\pi}_{R}\left(Q_{1}\right)=\hat{\pi}_{R}\left(Q_{2}\right)([12$, Corollary 3.7.2]). Moreover, we separate the cases $R \in C^{*} \backslash \operatorname{Sing}\left(C^{*}\right), R \in \mathbb{P}^{2 *} \backslash C^{*}$ and $R \in \operatorname{Sing}\left(C^{*}\right)$.

Firstly we suppose that $R$ is an inner Galois point, that is $R \in C^{*} \backslash \operatorname{Sing}\left(C^{*}\right)$. Then, the projection $\hat{\pi}_{R}$ is of degree five. Then, any ramification index is five, by the property mentioned above. Therefore, the set $\hat{\pi}_{R}^{-1}\left(\hat{\pi}_{R}(Q)\right)=\{Q\}$ if $Q \in \operatorname{Flex}(C)$. Since this condition does not coincide with (1), ..., (4) we have a contradiction.

Then we assume that $R$ is an outer Galois point, that is $R \in \mathbb{P}^{2 *} \backslash C^{*}$, so that $\operatorname{deg} \hat{\pi}_{R}=6$. Note that there exist no tangent lines with two contact points for $C^{*}$, by the smoothness of $C$ and projective duality. By the property 
of Galois extensions, any line containing $R$ and a singular point must satisfy $\left(1^{*}\right)$. Since the number of singular points is nine, there exists at most three lines containing $R$ and satisfying ( $\left.1^{*}\right)$. Applying Hurwitz formula to the projection $\hat{\pi}_{R}$, we have that the ramification divisor must have degree 12. Thus there exists another ramification point which is not a flex of $C$, but this contradicts the property of Galois extensions.

Finally, we assume that $R \in \operatorname{Sing}\left(C^{*}\right)$. Let $R_{0} \in \operatorname{Flex}(C)$ with $\gamma\left(R_{0}\right)=R$. By the property of Galois extensions, any line containing $R$ and another singular point must satisfy $\left(1^{*}\right)$. Let points $P \in \mathbb{P}^{2}$ and $Q_{1}, Q_{2}, Q_{3} \in \operatorname{Flex}(C)$ satisfy $P \in T_{Q_{i}} C$ for any $i$, as in condition (1). We may assume that $P=(1: 0: 0), Q_{1}=(0: 1: 0), Q_{2}=(0: 0: 1)$ and $C$ is defined by

$$
X^{3}+G_{1}(Y, Z) X^{2}+G_{2}(Y, Z) X+G_{3}(Y, Z)=0
$$

where $G_{i}$ is a homogeneous polynomial of degree $i$. Since $Q_{1}, Q_{2} \in \operatorname{Flex}(C)$ and $T_{Q_{1}} C$ (resp. $T_{Q_{2}} C$ ) is defined by $Z=0$ (resp. $Y=0$ ), $G_{1}=0$ and $G_{2}, G_{3}$ is divisible by $Y Z$. Since $T_{Q_{3}} C$ is defined by $\alpha Y+\beta Z=0$ for some $\alpha, \beta \in K \backslash 0$, we also have $G_{2}=0$. We have an equation $X^{3}+G_{3}(Y, Z)=0$. We can take a linear transformation $\phi$ of $\mathrm{P}^{2}$ such that $\phi(P)=P$ and the three points given by $X=G_{3}(Y, Z)=0$ move to $(0: 1:-1),(0: 1: \omega)$, $\left(0: 1: \omega^{2}\right)$ by $\phi$, where $\omega$ is a cubic root of -1 different from -1 . Then, $\phi(C)$ is given by $X^{3}+\alpha\left(Y^{3}+Z^{3}\right)=0$ for some $\alpha \in K$. We may assume that $C$ is defined by $X^{3}+Y^{3}+Z^{3}=0$. Note that the nine flexes are contained in the union of lines $X=0, Y=0$ and $Z=0$. For this Fermat curve, if $P_{1}, P_{2} \in$ Flex $(C)$, then there exists a linear transformation $\psi$ such that $\psi\left(P_{1}\right)=P_{2}$ and $\psi(C)=C$. Therefore, we may assume that $R_{0}=$ $(0: 1:-1)$. The dual map is given by $\left(X^{2}: Y^{2}: Z^{2}\right)$. We find that $\gamma(\mathrm{Flex}(C) \cap\{X=0\})=C^{*} \cap\{U=0\}$, and analogous equalities holds for the pairs $Y, V$ and $Z, W$. Here, we have $R=(0: 1: 1)$. Let $R^{\prime}=(1: 0: 1)$ and let $\ell$ be the line passing through $R, R^{\prime} \in \operatorname{Sing}\left(C^{*}\right)$, which is defined by $U+V-W=0$. Since $R \in C^{*} \cap\{U=0\}, R^{\prime} \in C^{*} \cap\{V=0\}$ and $\ell$ satisfies condition $\left(1^{*}\right)$, we have that $\ell$ must meet $C^{*}$ at another singular point lying on the line $\{W=0\}$. Thus we have a contradiction as $\ell$ intersects $\{W=0\}$ at $(1:-1: 0) \notin \operatorname{Sing}\left(C^{*}\right)$.

\section{Curves with extendable Galois points}

Before proving Theorem 1.4, we present a preliminary lemma involved in the proof. 
Lemma 4.1. Let $G(Y, Z), H(V, W)$ be homogeneous polynomials of degree d. Assume that $d^{d}(-G)^{d-1}=-H\left(G_{Y}, G_{Z}\right)$ holds and $m \geq 1$ is the maximal number such that $Y^{m}$ divides $G$. Then, $W^{d-m}$ divides $H$.

Proof. Let $G=Y^{m} F$. Then $F$ is not divisible by $Y$ and, by Euler formula $(d-m) F=Y F_{Y}+Z F_{Z}, F_{Z}$ also is not. Then, $G_{Y}=m Y^{m-1} F+$ $Y^{m} F_{Y}=Y^{m-1}\left(m F+Y F_{Y}\right)$ and $G_{Z}=Y^{m} F_{Z}$. We denote by $H=$ $\sum_{i=0}^{d} \alpha_{i} V^{i} W^{d-i}$. Then, by the assumption,

$$
\begin{aligned}
& d^{d}(-1)^{d-1} Y^{m(d-1)} F^{d-1}=-H\left(G_{Y}, G_{Z}\right) \\
= & -\sum_{i} \alpha_{i} Y^{i(m-1)+m(d-i)}\left(m F+Y F_{Y}\right)^{i} F_{Z}^{d-i} .
\end{aligned}
$$

Since $\left(m F+Y F_{Y}\right)^{i} F_{Z}^{d-i}$ is not divisible by $Y$ for any $i$, we have $\alpha_{i}=0$ for any $i$ such that $m(d-1)>i(m-1)+m(d-i)$, i.e. $i>m$. Therefore, $H=\sum_{i=0}^{m} \alpha_{i} V^{i} W^{d-i}=W^{d-m} \sum_{i=0}^{m} \alpha_{i} V^{i} W^{m-i}$.

Proof of Theorem 1.4(I). The assertion $(2) \Rightarrow$ (1) is nothing but Lemma 2.5(2). We prove (1) $\Rightarrow$ (2). By using projective duality, we may assume that $d \geq d^{*}$. By Proposition 2.3 , we may assume that $P=(1: 0: 0)$ be an extendable outer Galois point, $C$ is given by

$$
X^{d}+G(Y, Z)=0,
$$

where $G(Y, Z)$ is a homogeneous polynomial of degree $d$, and any birational transformation $\sigma \in G_{P}$ is represented by $\sigma(X: Y: Z)=\left(\zeta^{i} X: Y: Z\right)$ for some $i$. By Lemma 2.4, we have an injection $G_{P} \hookrightarrow G[\bar{P}]$, where $\bar{P}=(1: 0: 0)$. Then, $d^{*}=d$ and $G_{P}=G[\bar{P}]$. Therefore $\bar{P} \in \mathbb{P}^{2 *}$ is extendable outer Galois for $C^{*}$. Furthermore, $C^{*}$ is given by

$$
U^{d}+H(V, W)=0,
$$

where $H$ is a homogeneous polynomial of degree $d$. Since the dual map $\gamma: C \rightarrow C^{*}$ is given by $\gamma=\left(d X^{d-1}: G_{Y}: G_{Z}\right)$, we have $\left(d X^{d-1}\right)^{d}+$ $H\left(G_{Y}, G_{Z}\right)=0$. Since $X^{d}=-G$ on $C$, we have an equation

$$
d^{d}(-G)^{d-1}+H\left(G_{Y}, G_{Z}\right)=0
$$

on $C$. Since the variable $X$ does not appear, this equation holds as polynomials. For a suitable system of coordinates, by Lemma 2.2, $G(Y, Z)$ is divisible by $Y Z$. Let $m \geq 1$ (resp. $n \geq 1$ ) be the maximal number such that $Y^{m}\left(\right.$ resp. $\left.Z^{n}\right)$ divides $G$. Note that $m+n \leq d$. Then, by Lemma $4.1, H$ is 
divisible by $V^{d-n} W^{d-m}$. Since $(d-n)+(d-m)=2 d-(n+m) \geq d=$ $\operatorname{deg} H$, we have $H=\alpha V^{d-n} W^{d-m}$ for some $\alpha \in K$. Therefore, $C^{*}$ is projectively equivalent to the curve given by $U^{d}-V^{e} W^{d-e}=0$ for some $e \geq 1$. The curve $C$ is also by Lemma 2.5(1).

Proof of Theorem 1.4(II). The assertion $(3) \Rightarrow$ (1) is nothing but Lemma 2.5. We prove $(1) \Rightarrow(2)$. Let $P$ be an extendable outer Galois point for $C$ and let $Q$ be an extendable inner Galois point for $C^{*}$. By Proposition $1.5, d^{*} \geq d$ and $d \geq d^{*}-1$. Then we treat the cases $d=d^{*}-1$ and $d=d^{*}$ separately.

Firstly, we assume that $d=d^{*}-1$. By Proposition 2.3, we may assume that $P=(1: 0: 0)$, a generator $\sigma \in G_{P}$ is represented by $\sigma(X: Y: Z)=$ $(\zeta X: Y: Z)$ and $C$ is given by

$$
X^{d}+G(Y, Z)=0 .
$$

By Lemma 2.4, we have an injection $G_{P} \hookrightarrow G[\bar{P}]$, where $\bar{P}=(1: 0: 0)$. Considering the orders of $G_{P}$ and $G[\bar{P}]$, we have $G_{P}=G[\bar{P}]$. Hence $\bar{P} \in C^{*}$ is smooth and extendable inner Galois. Considering the action, $C^{*}$ is given by

$$
U^{d} H_{1}(V, W)+H_{d+1}(V, W)=0
$$

where $H_{i}$ is a homogeneous polynomial of degree $i$. For a suitable system of coordinates, by Lemma 2.2, we may assume that $H_{1}=W$. We denote by $H_{d+1}=H$. Then $H$ is not divisible by $W$ as $C^{*}$ cannot contain lines, and also $H_{V}$ is not by Euler formula. Since the dual map $\gamma_{C^{*}}: C^{*}{ }_{--\rightarrow} C^{* *}=C$ is given by

$$
\begin{gathered}
\gamma_{C^{*}}=\left(d U^{d-1} W: H_{V}: U^{d}+H_{W}\right)=\left(d U^{d-1} W^{2}: W H_{V}: U^{d} W+W H_{W}\right) \\
=\left(d U^{d-1} W^{2}: W H_{V}:-H+W H_{W}\right)
\end{gathered}
$$

by using $U^{d} W=-H$, we have

$$
\left(d U^{d-1} W^{2}\right)^{d}+G\left(W H_{V},-H+W H_{W}\right)=0 .
$$

Since $U^{d} W=-H$ on $C^{*}$, we have an equation

$$
d^{d}(-H)^{d-1} W^{d+1}=-G\left(W H_{V},-H+W H_{W}\right)
$$

on $C^{*}$. Since the variable $U$ does not appear, this equation holds as polynomials. Let $G(Y, Z)=\sum_{i=0}^{d} \alpha_{i} Y^{i} Z^{d-i}$. Then, we have

$$
d^{d}(-H)^{d-1} W^{d+1}=-\sum_{i} \alpha_{i} W^{i} H_{V}^{i}\left(-H+W H_{W}\right)^{d-i}
$$


Since $H_{V}$ and $-H+W H_{W}$ are not divisible by $W, \alpha_{i}=0$ for any $i$. This is a contradiction.

Then we assume that $d^{*}=d$. By Proposition 1.5, we have an injection $G_{Q} \hookrightarrow G[\bar{Q}]$. Considering the orders, we have $G_{Q}=G[\bar{Q}]$. Hence $\bar{Q} \in C$ is smooth and extendable inner Galois. We have assertion (2).

Then it remains to prove (2) $\Rightarrow(3)$. Let $P$ be an extendable inner Galois point for $C$ and let $Q$ be an extendable inner Galois point for $C^{*}$. By projective duality, we may assume that $d \geq d^{*}$, and Proposition 1.5 assures that $d^{*} \geq d-1$. Hence we deal with the cases $d^{*}=d-1$ and $d^{*}=d$ separately.

Assume that $d^{*}=d-1$. By Proposition 1.5 and considering the orders, we have $G_{P}=G[\bar{P}]$. Then, $\bar{P}$ is extendable outer Galois for $C^{*}$. Similarly to the above discussion to prove $(1) \Rightarrow(2)$, we have a contradiction.

Therefore, we have $d^{*}=d$. By Proposition 2.3, we may assume that $P=(1: 0: 0)$ and $C$ is given by

$$
X^{d-1} Z+G(Y, Z)=0 .
$$

By Lemma 2.4, we have an injection $G_{P} \hookrightarrow G[\bar{P}]$, where $\bar{P}=(1: 0: 0)$. Considering the orders of $G_{P}$ and $G[\bar{P}]$, we have $G_{P}=G[\bar{P}]$. Therefore, $\bar{P} \in \mathbb{P}^{2 *}$ is extendable inner Galois for $C^{*}$. Furthermore, $C^{*}$ is given by

$$
U^{d-1}(\alpha V+\beta W)+H(V, W)=0,
$$

where $\alpha, \beta \in K$. Since the dual map $\gamma: C \rightarrow C^{*}$ is given by

$$
\begin{gathered}
\gamma=\left((d-1) X^{d-2} Z: G_{Y}: X^{d-1}+G_{Z}\right)=\left((d-1) X^{d-2} Z^{2}: Z G_{Y}: X^{d-1} Z+Z G_{Z}\right) \\
=\left((d-1) X^{d-2} Z^{2}: Z G_{Y}:-G+Z G_{Z}\right)
\end{gathered}
$$

by using $X^{d-1} Z=-G$, we have

$$
\left((d-1) X^{d-2} Z^{2}\right)^{d-1}\left(\alpha Z G_{Y}+\beta\left(-G+Z G_{Z}\right)\right)+H\left(Z G_{Y},-G+Z G_{Z}\right)=0 .
$$

Since $X^{d-1} Z=-G$ on $C$, we have an equation

$$
(d-1)^{d-1}(-G)^{d-2} Z^{d}\left(\alpha Z G_{Y}+\beta\left(-G+Z G_{Z}\right)\right)=-H\left(Z G_{Y},-G+Z G_{Z}\right)
$$

on $C$. Since the variable $X$ does not appear, this equation holds as polynomials. Let $H(V, W)=\sum_{i=0}^{d} \alpha_{i} V^{i} W^{d-i}$. Then, we have $(d-1)^{d-1}(-G)^{d-2} Z^{d}\left(\alpha Z G_{Y}+\beta\left(-G+Z G_{Z}\right)\right)=-\sum_{i} \alpha_{i} Z^{i} G_{Y}^{i}\left(-G+Z G_{Z}\right)^{d-i}$.

Since $G_{Y}$ and $-G+Z G_{Z}$ are not divisible by $Z, \alpha_{i}=0$ for any $i<d$. 
Therefore, $H(V, W)=c V^{d}$ for some $c \in K$. We have an equation

$$
U^{d-1}(\alpha V+\beta W)+c V^{d}=0
$$

for $C^{*}$. The dual curve $C^{*}$ is projectively equivalent to the curve given by $U^{d}-V^{d-1} W=0$. Therefore, $C$ is also by Lemma 2.5(1).

REMARK 4.2. In order to deal with Problem 1.1, the condition $P \notin \operatorname{Sing}(C)$ in the definition of inner Galois point is crucial. In particular, Theorem 1.4 fails to hold true as we extend the assertion to (non-extendable) outer Galois point for $C$ and Galois point lying on $\operatorname{Sing}\left(C^{*}\right)$.

For example, let $C \subset \mathbb{P}^{2}$ be curve defined by $(X+Y+Z)^{3}-$ $27 X Y Z=0$. Then $(1: 0: 0)$ is an outer Galois point for $C$ ([14, Example 2]). Since $C$ is a rational nodal curve with three flexes, the dual curve $C^{*}$ is a quartic plane curve with three singular double points. Thus $C$ is not a selfdual curve. However, any singular point $Q \in \operatorname{Sing}\left(C^{*}\right)$ is a Galois point for $C^{*}$ as the projection $\pi_{Q}: C^{*}-\rightarrow \mathbb{P}^{1}$ has degree two.

REMARK 4.3. According to [6], the set of all Galois points for the plane curve defined by $X^{d}-Y^{e} Z^{d-e}=0$ is equal to $\{(1: 0: 0),(0: 1: 0),(0: 0: 1)\}$ (if $d \geq 4$ ).

Acknowledgments. The authors are grateful to Professor Yoshiaki Fukuma for helpful comments. The authors thank Professor Hisao Yoshihara for informing a new result of him with Hayashi [6]. The authors also thank the referee for helpful advice.

\section{REFERENCES}

[1] E. Arbarello, M. Cornalba, P. A. Griffiths and J. Harris, Geometry of algebraic curves, Vol. I. Grundlehren der Mathematischen Wissenschaften 267, Springer-Verlag, New York, 1985.

[2] H. C. CHANG, On plane algebraic curves, Chinese J. Math. 6 (1978), 185-189.

[3] C. Ciliberto, Alcune applicazioni di un classico procedimento di Castelnuovo, Seminari di geometria, 1982-1983 (Bologna, 1982/1983), Univ. Stud. Bologna, Bologna, 1984, 17-43.

[4] S. FuKASAWA, Galois points for a plane curve in arbitrary characteristic, Proceedings of the IV Iberoamerican conference on complex geometry, Geom. Dedicata 139 (2009), 211-218.

[5] R. HARTSHORne, Generalized divisors on Gorenstein curves and a theorem of Noether, J. Math. Kyoto Univ. 26 (1986), 375-386. 
[6] H. Hayashi and H. Yoshinara, Galois group at each point for some self-dual curves, Geometry 2013 (2013), Article ID 369420, 6 pages.

[7] S. L. Kleiman, Tangency and duality, In: Proceedings of the 1984 Vancouver conference in algebraic geometry, CMS Conference Proceedings, 6, Amer. Math. Soc., Providence, RI, 1986, pp. 163-226.

[8] K. MiuRA, Galois points for plane curves and Cremona transformations, J. Algebra 320 (2008), 987-995.

[9] K. Miura and H. Yoshinara, Field theory for function fields of plane quartic curves, J. Algebra 226 (2000), 283-294.

[10] M. NAMBA, Families of meromorphic functions on compact Riemann surfaces, Lecture Notes in Math. 767, Springer-Verlag, Berlin, 1979.

[11] M. NAmBa, Geometry of projective algebraic curves, Marcel Dekker, Inc., New York, 1984.

[12] H. Stichtenoth, Algebraic function fields and codes, Universitext, SpringerVerlag, Berlin, 1993.

[13] H. Yoshinara, Function field theory of plane curves by dual curves, J. Algebra 239 (2001), 340-355.

[14] H. Yoshinara, Galois points for plane rational curves, Far East J. Math. Sci. 25 (2007), 273-284.

[15] H. Yoshihara, Rational curve with Galois point and extendable Galois automorphism, J. Algebra 321 (2009), 1463-1472.

Manoscritto pervenuto in redazione il 29 Novembre 2012. 